\title{
85965 - APLICABILIDADE DE INSTRUMENTOS DE AVALIAÇÃO DA QUALIDADE DA DIETA PARA LONGEVOS: ESTUDO AMPAL
}

\author{
Pôster - Gerontologia
}

Valéia laniski / laniski,VB / Ponti'ficia Universidade Católica do rio Grande do Sul; Claudia Oliveira Safian / SAFIAN, C. A. O. / Ponti'ficia Universidade Católica do rio Grande do Sul; Angelo Jose Goncalves Bós / BOS, A. J. G. / Ponti'ficia Universidade Católica do rio Grande do Sul

Introdução: Estudos têm apontado à relação da qualidade da dieta e a longevidade. Entretanto, poucos instrumentos foram desenvolvidos para a avaliação epidemiológica da qualidade da dieta e, em menor número, específicos para as pessoas idosas ${ }^{1,2}$. Objetivo: Comparar os resultados do questionário de avaliação dos hábitos alimentares utilizado pela Pesquisa Nacional de Saúde (PNS, 2013) com os resultados do Questionário do Guia de Alimentação Saudável (QGAS)2 em longevos. Método: Estudo transversal, no qual foi utilizado questionário de hábitos alimentares da PNS (aplicado por entrevistadores não necessariamente profissionais da saúde) e o QGAS, elaborado pelo Ministério da Saúde em 2005 (aplicado por nutricionistas). Os instrumentos foram aplicados junto ao domicílio do idoso (90 anos e mais). Esta pesquisa foi aprovada pelo Comitê de Ética no 1842373, CAEE 60894416500005336. Resultados: Cada item alimentar da PNS foi pontuado conforme os critérios do QGAS, gerando uma pontuação geral. Observou-se que a pontuação geral da PNS apresentou uma relação significativa $(\mathrm{p}<0,001)$ com coeficiente de correlação de $26 \%$ com a pontuação geral do QGAS, quando aplicada por equipe multiprofissional. A pontuação geral da PNS apresentou um coeficiente de correlação de 34\%, com a pontuação geral do QGAS quando aplicada por profissional nutricionista. Conclusão: Conclui-se que o questionário dos hábitos alimentares da PNS pode ser usado na avaliação da qualidade da dieta em longevos, sendo mais fidedigno com a realidade quanto aplicado por nutricionistas.

Palavras- chave: Idoso. Comportamento alimentar. Inquéritos Epidemiológicos.

Referências: 1. Campos MTFS, Monteiro JBR, Ornelas APRC. Fatores que afetam o consumo alimentar e a nutrição do idoso. Rev Nutr. 2000, v.13(3):157-165; 2. Ministério da Saúde. Brasil. Secretaria de Atenção à Saúde. Guia alimentar para a população brasileira: promovendo a alimentação saudável. Brasília, 2008. 210 p. 